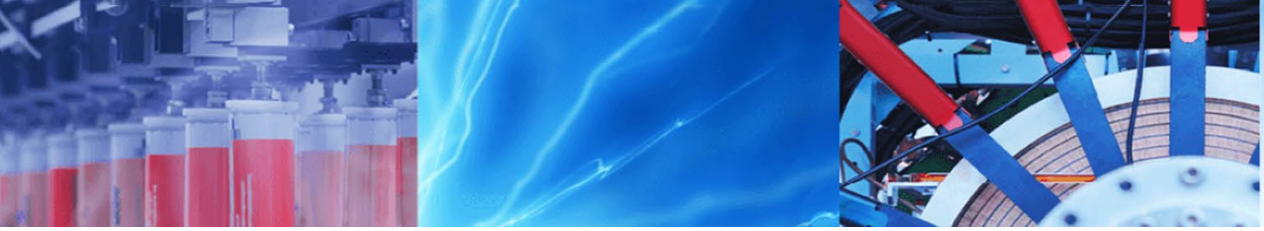

Research Article

\title{
Research on behavior modeling of emergency network rumor information dissemination processing
}

\author{
Hui Yuan ${ }^{1}$
}

Received: 11 May 2019 / Accepted: 19 November 2019 / Published online: 25 November 2019

(c) Springer Nature Switzerland AG 2019

\begin{abstract}
This study presents a complete behavioral model of network rumor information dissemination. Firstly, introduces the relevant psychological characteristics of communicator information processing behavior, such as media literacy, psychological distance, cognitive resources and stereotypes. Secondly, according to the research object, communicators are classified into event-related and event-independent kinds. The basic process of information dissemination of network rumors is proposed. After that, based on the combination of psychological model, statistical learning method and opinion dynamics, the event-related and event-independent rumors communicators behaviors are quantitatively modeled. This is a preliminary study combined with psychology model and statistical learning methods, the model comes from the data and has high credibility real-time dynamics, it can be used to predict the future evolution process of network rumor, which is of great theoretical significance and practical value.
\end{abstract}

Keywords Network rumor · Communicators · Behavior modeling

\section{Introduction}

At present, the research of public opinion communication are mainly based on statistical physics to analyze the essence of public opinion, and provide a basis for quantitative public opinion evolution modeling [1-3]. These models view individual netizens as a microscopic element and observe the overall macroscopic phenomenon by setting simple interaction rules. This method is still persuasive in the statistical study of online public opinion. But people are not a simple element, and the interaction between people is not just a simple rule. A new research method is needed, which can understand the law of the evolution of public opinion in essence, and it can also be consistent with the evolutionary effect of public opinion on the macro level.

Psychology models attempt to explain the psychological role in social behavior. These research are relatively early, different models are even contradictory. Many models only stay at the macro concept level. Statistical learning method has developed rapidly and is widely used in recent years. Its main function is to select the optimal model and train the optimal parameters, and it cannot provide the model needed to solve the problem directly. The opinion dynamics draw references from the methods in statistical physics, and the individual is seen as a basic element, the advantage is that the rules are simple, but the disadvantage is that it cannot reflect complex realities.

The combination of psychological model, statistical learning methods and opinion dynamics to take the advantages of the three models and make up for the shortcomings of the models. For the psychological model, it is necessary to integrate the psychological model related to the research, and to refine and quantitatively describe the psychological model; for the statistical learning method, the model that can solve the problem should

Hui Yuan, yuanhui.hunnu@foxmail.com | ${ }^{1}$ School of Journalism and Communication, Hunan Normal University, Changsha 410081, Hunan, People's Republic of China. 
be established; opinion dynamics is often used to build rougher netizen model. Since some concepts in psychology are very broad, there is no unified definition yet. Therefore, based on the research background of Network Rumor, this paper uses the unique psychological concept combined with Network Rumor to avoid confusion.

\section{Network rumor communicator information processing behavior characteristics}

In the process of rumor communicator processing information, it is not without rules, but has certain psychological laws. Related psychological characteristics, including media literacy, psychological capacity, psychological distance and stereotype.

\subsection{Media literacy characteristics of the communicator}

The definition of media literacy in the American Media Literacy Research Center in 1992 was that: Media literacy is the ability of people to choose, understand, question, evaluate, create, produce, and think about the various media information [4]. After that, many scholars have defined media literacy in different background $[5,6]$. But no matter how to define it, there are some common characteristics [6]. One is Media Coverage. Media literacy is the ability of individuals to integrate into social media, the Internet and other media. The existence of the media environment is the premise of media literacy. Middle-aged and older people may be more under the coverage of traditional media, while young people are more surrounded by the Internet. The other is Media Capabilities. Media literacy emphasizes the ability to identify information. Initially, media literacy was designed to describe the ability of adolescents to discern the influence of vulgarity. Today, in addition to the traditional listening, speaking, reading and writing abilities, media literacy should include the ability to collect, extract, interpret, evaluate, and critically absorb and disseminate various kinds of information.

So far, there is no unified definition of media literacy. According to the common characteristics of various definitions and the reality problems faced by, media literacy is defined as the information reception ability, information cognition ability and information dissemination ability of the Microblog information. First of all, Microblog is a form of media, which is a platform for people to obtain information from the Internet. This research focuses on the Microblog environment. Secondly, it refines the information processing capability and divides it into three parts: Information reception ability representative whether the communicator can receive the Microblog information and emphasize the role of the external network environment. If the communicator follows a certain netizen, then he can receive the information sent by the netizen; the Information cognitive ability indicates that whether the communicator is willing to and how to process information, reflects the subjective influence. Although information is received, Microblog information may not be processed due to factors such as time and energy. Information dissemination ability means that the communicator has the means to disseminate his attitude and opinion, which is also determined by the external network environment.

\subsection{Psychological distance characteristics of the communicator}

Psychological distance refers to a kind of subjective judgment that people appear in the heart according to the distance between the research object and the reference point [7]. The reference point mainly refers to oneself at this time and here. That is to say, the psychological distance emphasis more on oneself, and can be divided into time distance, spatial distance, social distance and hypothetical distance [8]. The time distance indicates the distance between things and oneself in the time dimension. The spatial distance indicates the distance between things and oneself in the geographical distance. The social distance indicates the relationship between people. The hypothetical distance is the possibility of the event in the real society.

In this research, the psychological distance focuses on emergencies, rather than a specific event. The information processing model of the communicator judges the psychological distance between the emergency and oneself mainly by the posting status on Microblog platform. If there are more discussions about the emergency, then the psychological distance is closer, the more likely it is to deal with the next emergency. If the number of emergencies involved is less, the farther the emergency is from oneself, and the possibility of handling the next emergency is less. Therefore, the psychological distance not only indicates the relationship between the emergency and oneself, but also the willingness to express.

\subsection{Cognitive resource characteristics of the communicator}

Cognitive resource theory refers to the energy people need in cognitive activities. Based on psychological resources, people can successfully complete various cognitive activities, such as controlling attention, updating memory, and regulating emotions [9]. Psychological resources are energy that regulates one's own state and 
provides support for various conscious psychological activities and external behaviors. In the real world, there is no quantitative modeling method for cognitive resources, but a macro concept.

This research attempts to quantitatively describe the cognitive resource in a specific environment, focus on the Microblog platform, and define cognitive resources as the possibility the communicator deal with emergency Microblog rumor information which is closer to oneself at a certain moment. If the cognitive resource is larger, the probability of handling the Microblog information of the emergency is higher; otherwise, the probability is lower. The difference between psychological distance and psychological distance is that psychological distance pays more attention to the relationship between things and oneself, while cognitive resources pay more attention to whether there is energy to deal with things. People's psychological distance and cognitive resources are changing with time. The psychological distance of things and the cognitive resources of dealing with things are different in different periods. But in general, the psychological distance does not change much in a short period of time, while the cognitive resources are reversed and there is a large fluctuation in a short time.

\subsection{Stereotype characteristics of the communicator}

In 1922, the famous journalist Walter Lippmann used stereotypes to explain people's misconceptions and prejudices, especially those that were incorrect and stubborn, that is, specific stereotypes [10]. Stereotypes tend to be strong and hinder our understanding of unknown objects. But stereotypes help people streamline their cognitive activities in order to speed up their work [11]. Stereotypes have a significant impact on cognitive activity processes. The role of stereotypes is to reduce unnecessary mental work, or to increase the use of cognitive resources. There are three main models for the impact of stereotypes on human cognitive activities: Schema filter model [12], Association network model [13] and Flexible coding model [12]. Some scholars have studied the main influences and countermeasures of stereotypes in the spread of public opinion [14-16].

This research draws references from the concept of stereotypes and gives meaning to its specific environment. It believes that stereotypes are the historical attitudes of netizens in the process of processing Microblog information. Historical experience will affect the current state to some extent, so one's previous attitude towards an emergency will have a certain impact on the current attitude.

The existing psychology study is only qualitative descriptions of certain aspects of the individual, not modeled in quantitative terms, and also the concepts are not the same. In this paper, for the psychological model, on the one hand, it is necessary to integrate the psychological model related to the research, on the other hand, the psychological model is refined and quantitatively described.

\section{Network rumor communicator classification and basic information dissemination process}

Based on the analysis of the behavioral characteristics of communicators' individual processing, this section quantitatively describes the individual behavior of communicator. First, this section divides communicators into event-related communicators and event-independent communicators; secondly, describes the individual process model of information dissemination of communicators.

\subsection{Rumor communicator classification}

Usually, the netizens can be classified according to individual attributes such as hobbies, personality traits, age and gender; they can also be classified according to social influence, such as opinion leaders or ordinary users; they can also be classified by community groups or network platforms, such as WeChat, Microblog and so on.

The subject of this research is the evolution of network rumors caused by emergencies. Therefore, this research classifies the communicator by the extent to which the communicator participates in the discussion of emergencies. If the communicators participate in the discussion of emergencies on the Microblog platform, the communicators are classified into event-related types, and vice versa, both the event-related and event-independent communicators are Microblog users.

For event-related communicators, since they know the time, content and behavior of the posts sent by the emergencies, then can extract individual behaviors such as psychological characteristics, personality characteristics and behavioral rules according to the published information, such communicators are the main emphasis of our research; for event-independent communicators, because cannot know their opinions, attitudes and emotions about the event, and cannot extract their characteristics attributes for emergencies, so it is difficult to study in depth.

\subsection{The basic process of Network rumor information dissemination}

Network rumors are reflected in the evolution of the number and attitude of Microblog posts in emergencies. The network rumor information dissemination can be divided into three parts: information, relationship networks and 
communicator individualizers. Generally speaking, after an emergency breaks out, the official media or the Network $\mathrm{V}$ (often refer to the opinion leader) grasps the information for the first time. They often process the information according to their own needs and disseminate the processed information to other netizens; the information is disseminated through many online network channels to the netizens, which include major Web Sites, Network Platforms, and Instant Message software; the communicator receives the information and decide whether to disseminate the information after processing the information. This continues to cycle all over again until the rumor completely disappears. As shown in Fig. 1.

\section{Network rumor information processing behavior model}

\subsection{Event-independent communicator behavior modeling}

This section combines the media literacy characteristics and opinion dynamics models of netizens to model eventindependent communicators.

\subsubsection{MR model}

The MR model is the abbreviation of Majority Rule, that is based on the principle of local maximization, which is used to study public policy issues such as elections. The basic idea of the MR model is that, the opinions of a few people should be subject to the opinions of most people within a group. The specific interaction rules are shown as below.

Step 1 Give each individual an opinion value $S_{i}$, and the opinion value of $S_{i}=+1$ or $S_{i}=-1$.

Step 2 In each step, $r$ individuals are randomly selected into a group, and each individual in the group uses the opinions of most individuals in the group. The size $r$ of the group is not fixed and can follow a certain distribu- tion. If $r$ is an odd number, then there will be majority opinions; if $r$ is an even number, then need to add an opinion artificially, such as adding $S_{i}=+1$ when counting the positive and negative opinions for each step.

In which, the number of groups is $N$, the number of each individual is $i(1 \leq i \leq N), r \leq N$, and the opinion of each individual is recorded as $S_{i}$.

\subsubsection{Information processing model based on opinion dynamics}

Based on the media literacy characteristics of the psychological model, the process of event-independent communicator processing information is divided into three stages: information reception, information cognition and information dissemination.

Information reception Information can be received from the netizens concerned and other sources. If the netizens that the communicator concerned have posted, then communicator can read the posts sent by other netizens; otherwise, they cannot read them.

Information cognition The method of opinion dynamics is used to simulate the event-independent communicator's information processing behavior and the attitude. Since the opinion of this model is a binary opinion, this study uses the MR model in the discrete-opinion dynamics model. In MR models, a person's opinion is determined by the people around him. That is, if the surrounding people have more positive opinions about an event than negative ones, then it can be speculated that this person is more likely to hold a positive opinion; if the surrounding people hold more negative opinions than positive ones, then the person is more likely to hold a negative opinion, as shown in Fig. 2.

Information dissemination Since event-independent communicators do not publish statements related to emergencies, they only receive information and do not publish information.
Fig. 1 The basic process of network rumor information dissemination

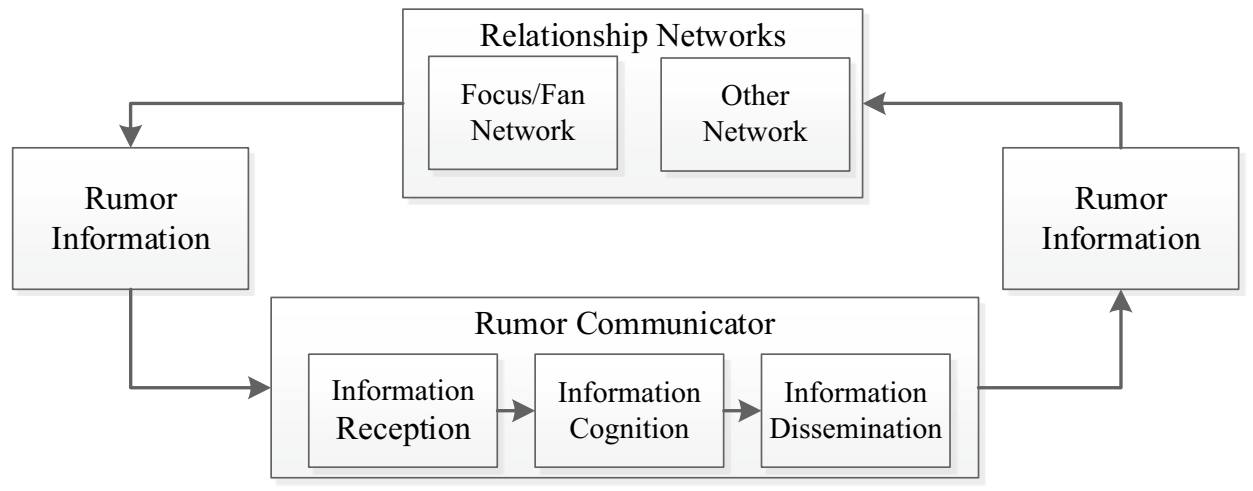




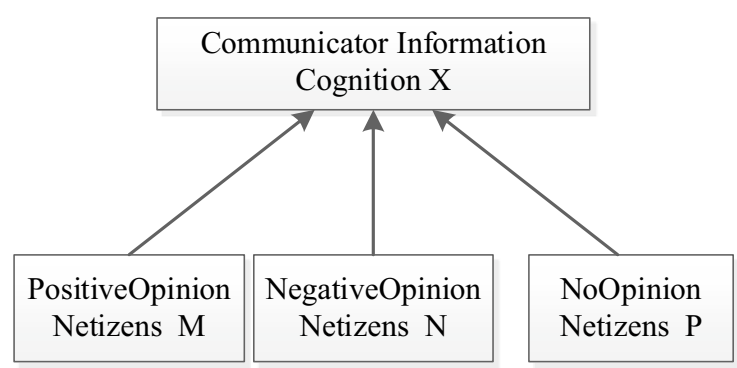

Fig. 2 Schematic diagram of the decision process of the eventindependent passer

\subsection{Event-related communicators behavior modeling}

This section mainly models the event-related communicators behavior.

\subsubsection{Overall framework}

The logic process of event-related communicators information processing behavior is shown in Fig. 3. When an event-related communicator processing information, he first determines whether he can receive the information. If the netizens concerned have published the information, the information can be disseminated to the netizen through the Microblog's focus/fan network, and vice versa. After that, the communicator judges whether to process the information according to the distance between the information and his psychological distance and the current cognitive resources. If the communicator processes the information, then determine the attitude of the post made at that moment according to the historical attitude to such events, the attitude of other netizens to such events, and the attitudes that they have expressed.

In the information reception phase, the event-related communicator is consistent with the event-independent messenger information processing behavior. In the information dissemination stage, information dissemination mainly describes its ability to disseminate information. This is different from whether it is willing to disseminate. Instead, it pays more attention to disseminating information on the platform. The main function is to disseminate the information that integrates its own attitudes to its fans.

\subsubsection{Judgment model based on psychological characteristics}

4.2.2.1 Psychological distance calculation of the communicator If the communicator is not interested in something and has no interest relationship, that is to say, the psychological distance is very far from itself, then the probability that the communicator process the information is very low; otherwise, it is very high. For network rumors, if one doesn't make a statement on the Internet, you can't extract the psychological distance from the network data, and it will not have any impact on other Internet users and even network rumors. In order to characterize the psychological distance that can influence the network rumors, that is, including the distance from the emergency to one-

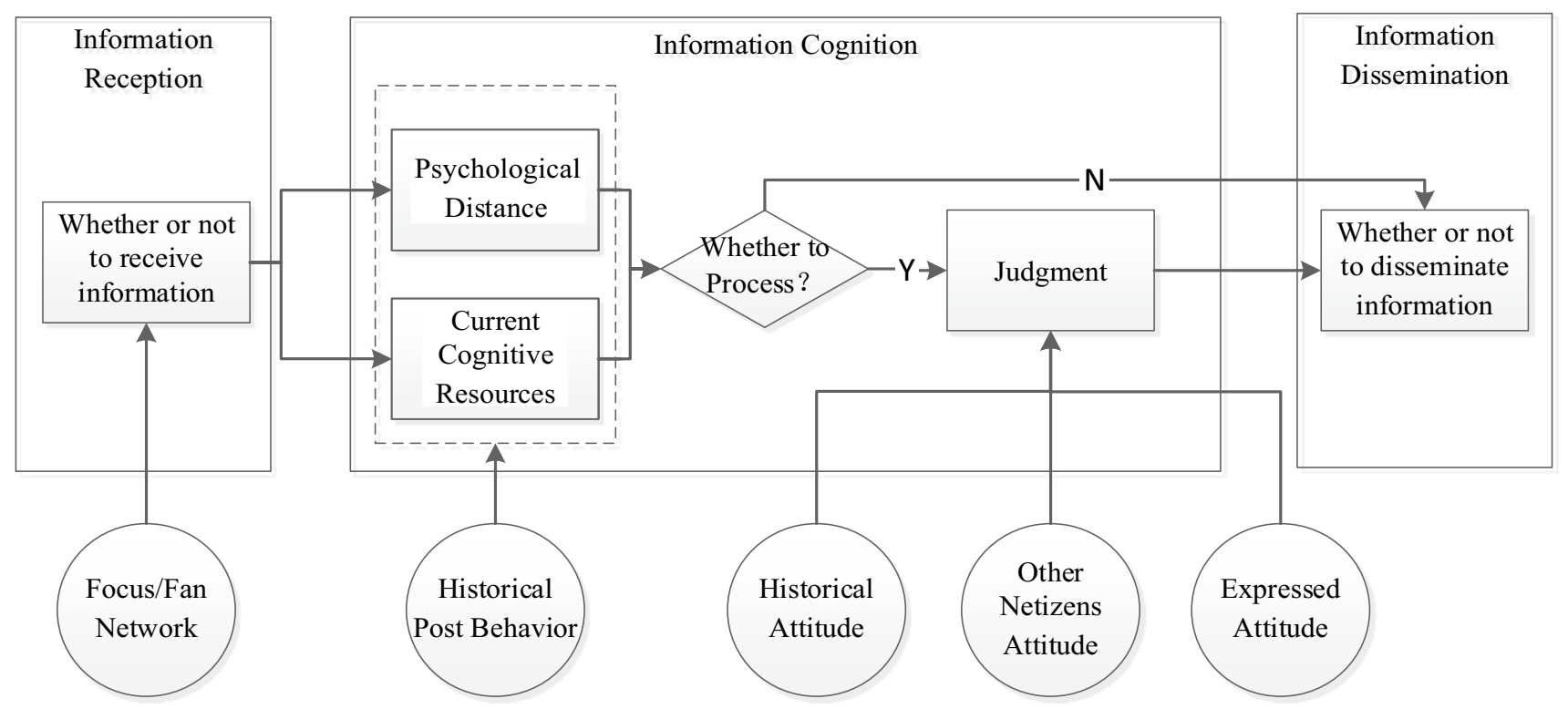

Fig. 3 Event-related communicator behavior model framework 
self and the willingness to express, define the psychological distance $P D$ of the communicator as:

$P D=P N / E N$

That is, the psychological distance is determined according to the posting behavior of the communicator on an event. In which, EN is the total number of emergencies, and $P N$ is the number of emergencies involved.

4.2.2.2 Cognitive resource calculation of the communicator According to the characteristics of the cognitive resources of the communicator, under the condition that the event is more relevant to oneself, the netizen will decide whether to process the information and disseminate the information according to the state of time and energy. If the communicator does not post, then there will be no impact on other netizens. Human cognitive resources are a quantity that changes with time, and is the probability that they will be willing to deal with the Microblog emergency after they first get the message. Define the communicator cognitive resource $C R(t)$ as follows:

$C R(t)=\sum_{r=1}^{M} P O_{r}(t) / M, t \in[0, N], P O_{r}(t) \in\{0,1\}$

In which, $C R(t)$ represents the cognitive resource at time $t ; M$ is the number of emergencies that the communicator participates in the discussion. If the communicator makes a speech in 10 emergencies, then $M=10 ; N$ is concerned time length of cognitive resources, if one follows the cognitive resources of netizens within 20 days, then $N=20$; $P O_{r}(t)$ is whether the communicator has expressed his opinions in the $r$ th emergency in $t$-time; $t$ is the moment knowing the event.

4.2.2.3 Process judgment In the process of judging whether to process the information, it is proposed to make the judgment in a form of probability. The specific rules are as follows:

$\left\{\begin{array}{l}f(p 1<P D \& \& p 2<C R(t)) \\ \text { then process. } \\ \text { else quit. }\end{array}\right.$

In which, $U[0,1]$ subject to uniform distribution of 0 to 1.

\subsubsection{Markov chain-based opinion decision model}

4.2.3.1 Markov chain model The Markov chain is a Markov process of discrete time and discrete states. The characteristic of the Markov process is that when the state of the random process at time $t_{0}$ is a known condition, the state at which the process is at time $t\left(>t_{0}\right)$ is only related to the state at time $t_{0}$. The previous state has nothing to do with it. This feature is called no aftereffect. That is, the future state of the process is only related to the current state and has nothing to do with the past.

The core idea of the Markov chain consists of two parts. First, the research object is modeled, that is, the state of the research object is extracted, and the transition relationship between the states is constructed; secondly, the probability of the transition relationship is calculated, and the sum of the outputs probabilities of the states is 1 .

4.2.3.2 Opinion decision based on Markov chain According to social influence theory, three elements will influence the formation of one's opinion, that are other opinions, one's own opinions, government and media opinions [17-19]. This research draws references from the social influence theory, and summarizes the factors that influence the formation of individual opinions into historical opinions, other people's opinions and their own published opinions, combine other opinions and their own opinions in social influence theory into other people's opinions. One's own opinions are divided into historical and published opinions. The opinions of others are external and can influence their own decision-making behavior.

The stereotype is the historical attitude of the communicator to the information on the emergency carried by Microblog. Stereotypes are equivalent to everyone's experience and can guide current decisions. Published opinions often have a direct impact on current opinions. Most people want to be able to persuade others to get themselves recognized, not to be convinced by others. Therefore, people will not easily deny what they have already said. The difference between published and historical opinions is that, historical opinions are netizens' attitudes towards other emergencies, and published opinions are netizens' attitudes toward the emergencies being studied.

This research divides the communicator's opinions decision process into two parts: processing information for the first time and processing information for the second time or later. There is no published opinion when processing information for the first time. The factors that influence the decision-making of the communicator are historical opinions and opinions of others. After the second processing of information, there are published opinions, and the factors that influence the decision-making of the communicator are historical opinions, opinions of others, and published opinions. At the same time, if the communicator publishes an opinion about the event, then that opinion is the published opinion about the event; if there is a subsequent 
published opinion, then the published opinion is updated with the new one.

Based on the above ideas, this research models the communicator's opinion decision based on the Markov chain. First, we abstract the three states of the communicator: historical opinions, other opinions, and published opinions, expressed by HP, OP, and PP, respectively. Among them, $\mathrm{HP}, \mathrm{OP}, \mathrm{PP} \in\{0,1\}, 0$ indicates a negative opinion, and 1 indicates a positive opinion. If the amount of positive information is greater than the negative information in all other people's information received by the communicator, then the opinion of others is positive, that is $O P=1$; otherwise is negative, $O P=0$. Similarly, if the number of positive information is greater than the amount of negative information in the historical opinion, $H P=1$; otherwise, $H P=0$. In general, historical attitudes do not change over time in a short period of time and are therefore defined as constant values. In the process of modeling the communicator using the Markov chain, two steps need to be completed, the first is to determine the form of state transition, and the second is to calculate the probability of state transition.

The state transition form when processing information for the first time is shown in Table 1.

Similarly, the state transition form when processing information for the second time and later is shown in Table 2.

After determining the state transition form, the probability of each situation is estimated by the idea of the maximum likelihood estimation. That is, if a certain parameter is known to make the sample have the highest probability of occurrence, then other less likely samples will not be selected. Therefore, this parameter is also used as the estimated true value. Therefore, the probability of each case is:

$I\left(\theta_{i}\right)=\frac{\left|\theta_{i}\right|}{\sum_{i=1}^{W}\left|\theta_{i}\right|}, \quad W=\{8,16\}$

In which, $\left|\theta_{j}\right|$ indicates the number of occurrences of the $\theta_{i}$ th case, $I\left(\theta_{i}\right)$ indicates the probability of occurrence of

Table 1 Processing information for the first time

\begin{tabular}{lll}
\hline$\theta_{i}$ & Transition condition & Transition result \\
\hline 1 & $H P=0, O P(t)=0$ & $P P(t+1)=0$ \\
2 & $H P=0, O P(t)=0$ & $P P(t+1)=1$ \\
3 & $H P=0, O P(t)=1$ & $P P(t+1)=0$ \\
4 & $H P=0, O P(t)=1$ & $P P(t+1)=1$ \\
5 & $H P=1, O P(t)=0$ & $P P(t+1)=0$ \\
6 & $H P=1, O P(t)=0$ & $P P(t+1)=1$ \\
7 & $H P=1, O P(t)=1$ & $P P(t+1)=0$ \\
8 & $H P=1, O P(t)=1$ & $P P(t+1)=1$ \\
\hline
\end{tabular}

Table 2 Second and subsequent processing information

\begin{tabular}{lll}
\hline$\theta_{i}$ & Transition condition & Transition result \\
\hline 1 & $H P=0, O P(t)=0, P P(t)=0$ & $P P(t+1)=0$ \\
2 & $H P=0, O P(t)=0, P P(t)=0$ & $P P(t+1)=1$ \\
3 & $H P=0, O P(t)=0, P P(t)=1$ & $P P(t+1)=0$ \\
4 & $H P=0, O P(t)=0, P P(t)=1$ & $P P(t+1)=1$ \\
5 & $H P=0, O P(t)=1, P P(t)=0$ & $P P(t+1)=0$ \\
6 & $H P=0, O P(t)=1, P P(t)=0$ & $P P(t+1)=1$ \\
7 & $H P=0, O P(t)=1, P P(t)=1$ & $P P(t+1)=0$ \\
8 & $H P=0, O P(t)=1, P P(t)=1$ & $P P(t+1)=1$ \\
9 & $H P=1, O P(t)=0, P P(t)=0$ & $P P(t+1)=0$ \\
10 & $H P=1, O P(t)=0, P P(t)=0$ & $P P(t+1)=1$ \\
11 & $H P=1, O P(t)=0, P P(t)=1$ & $P P(t+1)=0$ \\
12 & $H P=1, O P(t)=0, P P(t)=1$ & $P P(t+1)=1$ \\
13 & $H P=1, O P(t)=1, P P(t)=0$ & $P P(t+1)=0$ \\
14 & $H P=1, O P(t)=1, P P(t)=0$ & $P P(t+1)=1$ \\
15 & $H P=1, O P(t)=1, P P(t)=1$ & $P P(t+1)=0$ \\
16 & $H P=1, O P(t)=1, P P(t)=1$ & $P P(t+1)=1$ \\
\hline
\end{tabular}

the $\theta_{i}$ th case, and $W$ indicates whether the information is processed for the first time or the information is processed for the second time and later. $I\left(\theta_{i}\right)$ is one step away from the Markov transition probability, because the Markov transition probability is the probability that a certain outcome will occur under certain transition conditions. The $I\left(\theta_{i}\right)$ described is the probability that a certain transition condition and the transition result will co-occur. Then, the Markov chain transition probability is:

$p^{j}\left(\theta_{i}\right)=\frac{I\left(\theta_{i+j}\right)}{I\left(\theta_{i}\right)+I\left(\theta_{i+1}\right)}, \quad j \in\{0,1\}$,

$i=2 k-1, \quad k \in[1,2, \ldots, W / 2]$

In which, $p^{j}\left(\theta_{i}\right)$ is the probability when the state transition condition is $\theta_{i}$ and the transition result is $j$. As you can see from the formula, $p^{0}\left(\theta_{i}\right)+p^{1}\left(\theta_{i}\right)=1$.

\section{Discussion}

At present, the model of network rumor communicator in the previous research is simple, out of data and with poor predictive ability, and many psychological characteristics are only qualitative descriptions of certain aspects of the individual, not modeled in quantitative terms, and the concepts are also not the same. As the model in the current public opinion simulation is simple and difficult to predict the trend of network rumor, this paper proposes a "psychological-behavior" information processing model for rumors communicators, which is a preliminary study 
combined with psychology model and statistical learning methods.

This paper divides rumor communicators into eventrelated and event-independent types, and proposes a "psychological-behavior" model which can model the psychological mechanism affects the external behavior of the rumor communicator. Based on the theories of social psychology, statistical learning model and information dissemination model, the information processing model of event-related communicator is proposed. Based on the opinion dynamics, the information processing model of event-independent communicator is proposed.

The difference with the previous rumor communicators information processing model is that, on the one hand, this paper has a profound theoretical brace for modeling rumor communicator from the perspective of psychology. On the other hand, the model proposed in this paper adopts the method based on public opinion data modeling. The model comes from the data and has high credibility real-time dynamics. It can be used to predict the future evolution process of network rumor, which is of great theoretical significance and practical value.

In addition, what needs special attention is that, the verification and validation of model is very important. As models are simplifications of real systems. The granularity of the model determines the accuracy of the model. The boundary of the model determines the scope of application of the model. In the past, modeling of public opinion communication was often based on a set of logical structures hypothesis, and then simulated or logically derived the structure. This can be called mechanism simulation, but more work needs to be done before the actual application, that is, the model parameters must be combined with the actual meaning. Because of the lack of verification of empirical data, the reliability of the model is difficult to verify.

Therefore, in the future, network rumor information dissemination processing modeling should be based on data and combined with many previous mechanism modeling methods. A macroscopic idea is to combine more mature models in other fields, such as sociology, communication, psychology, game theory, Markov decision process and statistical learning theory, with the field of public opinion simulation, and then assigned values to the parameters in the model. A more ideal situation is that dynamic data drives modeling, that is to say, adding constantly updated data and correcting the parameters appearing in the model.

At present, data-based modeling is still in the exploration phase. As the emergence of public opinion monitoring systems, providing a large amount of data for public opinion research. However, the development of public opinion simulation is slow, on the one hand, because the research on the internal behavior mechanism of individual individuals is not thorough enough, and the research results in various fields are difficult to integrate; on the other hand, it is difficult to collect complete data of each person. Therefore, this is also a challenging direction in the field of public opinion modeling and simulation.

\section{Conclusion}

The research on network rumor communication mechanism is an important part of network rumor management, and the information processing behavior of network rumor communicators is the core of mechanism research. The psychological mechanism of rumor communicators is an important factor in triggering communication behavior. Communication behavior is external manifestation of psychological mechanism, the whole process can be described by "psychological-behavior". This paper studies the network rumor through the modeling of Microblog rumors communicator, and proposes a "psychologicalbehavior" information processing model. According to the degree of participation in the event, the rumor communicators are classified into two categories: event-related and event-independent. Based on social psychology, the behavior of rumor communicator to process information is divided into three stages: information reception, information cognition and information dissemination, and quantitatively constructing information processing behaviors model of event-related and event-independent rumors communicators.

Funding This research was supported by the Humanities and Social Sciences of Ministry of Education Planning Fund - Youth Fund (Grant No. 17YJC860032), and Hunan Province Social Science Fund Youth Project (Grant No. 16YBQ046).

\section{Compliance with ethical standards}

Conflict of interest I declare that I do not have any commercial or associative interest that represents a conflict of interest in connection with the work submitted.

\section{References}

1. Liu C, Hu X et al (2009) Research on network-based public opinion propagation model based on agent. Comput Simul 26(1):20-23

2. Li G (2016) Analysis of network public opinion trend based on community user characteristics. J Inf 35(9):93-98

3. Li Z (2009) Research on several algorithms in network public opinion communication. Beijing Jiaotong University, Beijing 
4. Aufderheide $P$ (1992) A report of the National leadership conference on media literacy. http://www.trans/uoregon/MediaLit/FA/ aufderheide/report.html

5. Li J (2017) New media literacy: concept and competence framework. J Zhejiang Commun Univ 24(2):15-19

6. Chen X, Wang X et al (2012) The evolution of American Media Literacy definition and the transformation of conference theme. China Electrochem Educ 7:19-22

7. Trope Y, Liberman N (2010) Construal-level theory of psychological distance. Psychol Rev 117(2):440

8. Hua S, Lu H (2012) The dimensions, relationship and related research area extensions of psychological distance. Adv Psychol 2(2):53-55

9. Su W, Ansheng W (2006) Cognitive psychology. Peking University Press, Beijing

10. Lu W (2010) A review of stereotype studies in journalism and communication. Yunmeng J 31(3):152-155

11. Fiske ST, Neuberg SL (1990) A continuum of impression formation, from category-based to individuating processes: influences of information and motivation on attention and interpretation. Adv Exp Soc Psychol 23:1-74
12. Sherman JW, Lee AY et al (1998) Stereotype efficiency reconsidered: encoding flexibility under cognitive load. J Pers Soc Psychol 75(3):589

13. Srull TK, Lichtenstein $M$ et al (1985) Associative storage and retrieval processes in person memory. J Exp Psychol Learn Mem Cogn 11(2):316

14. Niu Q (2015) Research on stereotypes in netizens' original news. Beijing University of Posts and Telecommunications, Beijing

15. Wu L, Wang C (2013) Analysis of the stereotype of labeled paradox in news communication. Editors' Assoc 11:59-61

16. Ling $H$ (2009) Negative influence of stereotype on national image communication and countermeasures. J Inf 11:57-58

17. Lewenstein M, Nowak A, Latané B (1992) Statistical mechanics of social impact. Phys Rev A 45(2):763

18. Schweitzer F, Holyst JA (2000) Eur Phys J B 17:723-729

19. Wang G, Liu Y et al (2017) Research on the alienation polarization effect of public opinion crisis. J Manag Sci 20(3):148-160

Publisher's Note Springer Nature remains neutral with regard to jurisdictional claims in published maps and institutional affiliations. 